\title{
The Roles of Sex, Innervation, and Androgen in Laryngeal Muscle of Xenopus laevis
}

\author{
Martha L. Tobias, Melanie L. Marin, and Darcy B. Kelley \\ Department of Biological Sciences, Columbia University, New York, New York 10027
}

\begin{abstract}
The relative contributions of innervation and androgen to three muscle fiber properties-twitch type, size, and number-were examined in the sexually dimorphic, androgensensitive laryngeal muscle of Xenopus laevis. In adults, the muscle contains all fast-twitch fibers in males and fast- and slow-twitch fibers in females; laryngeal muscle fibers are larger and more numerous in males than in females. Juvenile larynges are female-like in both sexes; male laryngeal muscle is subsequently masculinized by androgen secretion during postmetamorphic development. Because both laryngeal motor neurons and muscle fibers are androgen sensitive during masculinization, we examined the role of the nerve in androgen-regulated muscle flber development. Laryngeal muscle of male and female juvenile frogs was unilaterally denervated, and effects on muscle fiber type, size, and number were examined 4 weeks later. Half of the frogs received a dihydrotestosterone pellet at the time of denervation. Androgen treatment converts laryngeal muscle from mixed slow and fast to all fast twitch in both innervated and denervated muscle. Thus, the nerve is not required for androgen-regulated fiber type expression in either sex. Denervation produces muscle fiber atrophy and androgen treatment induces muscle fiber hypertrophy in male and female larynx. Nerve and hormone effects are independent and additive; fiber size in androgen-treated denervated muscle is greater than in untreated innervated muscle, and fiber size in androgentreated denervated muscle is smaller than in androgen-treated innervated muscle. There is no sex difference in the effects of innervation or androgen on fiber size. Denervation causes laryngeal muscle fiber loss in males but not in females. Androgen treatment protects male laryngeal muscle from denervation-induced fiber loss and causes fiber addition in innervated female laryngeal muscle. We conclude that there is a sexually dimorphic interaction between innervation and androgen in control of laryngeal muscle fiber number.
\end{abstract}

[Key words: dihydrotestosterone, trophic, myogenesis, denervation, twitch type, sexual dimorphism]

\footnotetext{
Received Apr. 29, 1992; revised July 20, 1992; accepted July 22, 1992.

We gratefully acknowledge John Robertson's assistance with preparation and photography of electron micrographs and William Gehrin's help with statistical analyses. We also thank Diana Catz, Michael Cohen, Leslie Fischer, and John Robertson for helpful comments on the manuscript. This work was supported by National Institutes of Health grants NS19949 and NS23684.

Correspondence should be addressed to Dr. Martha Tobias, Department of Biological Sciences, 909 Fairchild, Columbia University, New York, NY 10027. Copyright (C) 1993 Society for Neuroscience $0270-6474 / 93 / 130324-10 \$ 05.00 / 0$
}

Two extrinsic influences, innervation and circulating hormones, play important roles in muscle differentiation. Innervation is required for the normal development of muscle fiber twitch type and myosin isozyme expression; cross-innervation with a foreign nerve can induce conversion of fiber type even in adult muscle (reviewed in Jolesz and Streter, 1981). Innervation is also required during development for muscle growth and fiber addition (Fredette and Landmesser, 1991). In adulthood, denervation results in muscle atrophy (Harris, 1974). In hormonesensitive muscles, the endocrine milieu also influences muscle fiber growth and type. For example, the absence of thyroid hormone in rats results in a delay of adult myosin expression in hindlimb muscle and absence of adult myosin expression in cardiac muscle (Izumo et al., 1986). Androgenic hormones are essential for survival and growth of penile muscles in rats (Breedlove and Arnold, 1983) and for expression of specific myosin isoforms in arm muscles of frogs (Rubinstein et al., 1983) and jaw muscles of guinea pigs (Lyons et al., 1986). In guinea pig jaw muscles, castration induces fiber atrophy and expression of female typical myosins.

Although it is clear that both neural and hormonal factors influence muscle differentiation, the nature of their interaction in hormone target muscles is unclear. For example, can androgen treatment compensate for denervation, or are both required for full muscle differentiation? Here, we examine these issues in a highly androgen-dependent muscle, the dilator laryngis muscle of the frog Xenopus laevis. Laryngeal muscles effect song production and are markedly different in males and females. In adults, male laryngeal muscle is larger and contains twice as many muscle fibers as female laryngeal muscle (Sassoon and Kelley, 1986; Marin et al., 1990). Male laryngeal muscle is composed entirely of fast-twitch fibers, while female laryngeal muscle is composed of both fast- and slow-twitch fibers (Sassoon et al., 1987).

Sexual differentiation of the larynx occurs during postmetamorphic development, a period from the end of metamorphosis [postmetamorphic stage $0(\mathrm{PM} 0)$ ] to adult reproductive maturity (PM6; sec Tobias et al., 1991a). At PM0, laryngeal muscle size, fiber number, and fiber twitch type are the same in males and females (Sassoon and Kelley, 1986; Sassoon et al., 1987; Marin et al., 1990; Tobias et al., 1991a). Subsequently, the male larynx undergoes a profound transformation involving rapid growth, fiber addition, and conversion of fiber twitch type. In contrast, female larynx undergoes few changes during postmetamorphic development; growth and fiber addition are slow, and there is little change in fiber twitch type.

Masculinization of the $X$. laevis larynx is regulated by androgen secretion (Sassoon and Kelley, 1986; Sassoon et al., 1987; Marin et al., 1990; Tobias et al., 1991b). Castration completely 
arrests fiber type conversion and retards muscle growth and fiber addition. Testosterone treatment early in development completely converts male and female laryngeal muscle fibers to all fast twitch, speeds laryngeal growth in both sexes, and increases fiber addition in females (Marin et al., 1990; Tobias et al., 1991 h). Both developing laryngeal muscle and developing laryngeal motor neurons contain androgen receptor (Gorlick and Kelley, 1986; Kelley et al., 1989; Fischer and Kelley, 1991). Thus, androgen regulation of muscle differentiation could occur at the level of the muscle itself, at the level of the motor neurons that innervate the larynx or both.

In this article, we attempt to sort out the relative roles of nerve and hormone by examining hormone effects in denervated larynx. Specifically, we explore the ability of androgen to masculinize fiber twitch type, size, and number in denervated male and female larynges. Denervation was performed in juvenile frogs prior to masculinization of muscle fiber type and full attainment of a masculine fiber number.

\section{Materials and Methods}

\section{Animals}

Juvenile frogs at postmetamorphic stages 1-3 (PM1-PM3) were obtained from Nasco (Fort Atkinson, WI). Animals were staged by age and body weight as described in Tobias et al. (1991a); assignment to stage was confirmed by obtaining laryngeal weights from an additional one or two males in each group prior to treatment. Body and larynx weights at death are given in Table 1 . Frogs were maintained on a 12 $\mathrm{hr}: 12 \mathrm{hr}$ light/dark cycle in $0.4 \%$ saline in polycarbonate tanks at $21^{\circ} \mathrm{C}$ and fed frog brittle (Nasco) daily.

\section{Surgical procedures}

Each animal was deeply anesthetized in $0.1 \%$ MS 222 (ethyl 3-aminobenzoate methanesulfonic acid; Aldrich) prior to surgery. The frog was placed on its left side surrounded by ice with both front and rear limbs extended. A small incision was made to the right of the abdominal midline, one fat body and its attached gonad were extruded, and the sex of the frog was determined. The larynx was then exposed through an L-shaped incision that extended $1 \mathrm{~cm}$ posterior from the forelimb and continued medially to the abdominal midline. The underlying pectoralis muscle and portions of the external abdominal oblique muscle were incised in the same manner. Care was taken to avoid the subclavian artery and the anterior abdominal vein. Skin and muscle were retracted to expose the fatty tissue and cartilage surrounding the larynx. These tissues were grasped and gently extruded to bring the attached larynx into view. The right posterior end of the larynx was separated from the cartilaginous wing to which it is attached. The laryngeal nerve, visible extending from the posterolateral edge of the larynx, was dissected out while retracting the cartilaginous wing. The nerve was separated from the connective tissue encasing it and at least $4 \mathrm{~mm}$ excised to prevent reinnervation during the 4 week survival period. The larynx was returned to its anatomical position and muscle and skin were sutured. Frogs were allowed to recover in moist towels and then placed in $0.4 \%$ saline.

Masculinization of fiber type normally occurs between PM2 and PM5 (i.e., between 6 and 12 months after metamorphosis is complete; Tobias et al., 1991a). Examining fiber type switching in denervated larynges at the end of this extended period might have been complicated by the reestablishment of innervation or by the loss of substantial numbers of muscle fibers. We thus chose to treat acutely with a supraphysiological dose of androgen that had been shown to masculinize fiber type completely in PM2 frogs within 5 weeks (Tobias et al., 1991b). Immediately after denervation, while still on ice, half of the animals received an implant of 5-6 mg of dihydrotestosterone (DHT; Sigma) compressed into a 3-mm-diameter pellet and coated in Silastic polymer (Dow Corning, \#783 or \#891). The pellet was inserted through a small incision into the dorsal lymph sac. Dihydrotestosterone is highly soluble in the Silastic coating, which prevents pellet loss by blunting the sharp edges of the hormone pellet. At death 4 weeks later, the frog was deeply anesthetized by immersion in $0.1 \%$ MS 222 . The larynx was then carefully examined, using a dissecting microscope, for signs of nerve regen-

\begin{tabular}{|c|c|c|}
\hline Group & $\begin{array}{l}\text { Body weight } \\
\text { (gm) }\end{array}$ & $\begin{array}{l}\text { Larynx weight } \\
\text { (mg) }\end{array}$ \\
\hline \multicolumn{3}{|l|}{ Fiber type } \\
\hline Female & $7.4 \pm 1.0$ & $33 \pm 8$ \\
\hline Male & $7.8 \pm 0.6$ & $74 \pm 40$ \\
\hline Female + DHT & $11.4 \pm 2.8$ & $175 \pm 14$ \\
\hline Male + DHT & $8.0 \pm 0.9$ & $240 \pm 23$ \\
\hline \multicolumn{3}{|c|}{ Fiber size and number } \\
\hline Female & $7.7 \pm 2.0$ & $30 \pm 7$ \\
\hline Male & $7.4 \pm 1.0$ & $76 \pm 34$ \\
\hline Female + DHT & $8.8 \pm 1.8$ & $146 \pm 30$ \\
\hline Male + DHT & $8.0 \pm 1.2$ & $244 \pm 36$ \\
\hline
\end{tabular}

Values are given as means $\pm \mathrm{SD} . N=6$ for all groups.

eration (i.e., attachment of visible nerve fasicles); it was then removed and weighed.

\section{Histological procedures}

Fiber typing. Six animals from each treatment group were used. Each larynx was quickly frozen in liquid nitrogen and sectioned $(18 \mu \mathrm{m})$ transversely on a cryostat (Hacker). The sections were processed according to a modified acid-stable ATPase staining method (Guth and Samaha, 1970), which distinguishes fast- and slow-twitch fibers in $X$. laevis laryngeal muscle (Sassoon et al., 1987; Tobias et al., 1991a). Preincubation at $\mathrm{pH} 4.6$ and incubation at $30^{\circ} \mathrm{C}$ were found to be optimal. The percentage of slow-twitch fibers was determined for each larynx as described previously (Tobias et al., 1991a).

Fiber size and number. Each larynx was fixed in fresh $4 \%$ paraformaldehyde for at least $18 \mathrm{hr}$, postfixed with $1 \%$ osmium tetroxide in $0.1 \mathrm{M}$ sodium cacodylate buffer for $1-3 \mathrm{hr}$, and embedded in Epon (LX112, Ladd). Larynges were then transversely sectioned (5-7 $\mu \mathrm{m})$ on a rotary microtome (Spencer 820, AO). From each larynx, three sections with the greatest muscle area were identified as described previously (Marin et al., 1990). The total cross-sectional arca of the muscle was determined either by weighing projected tracings of the cross sections or by measuring the traced cross sections on a computer-interfaced bit pad (Sigma Scan, Jandel Scientific; procedure as in Marin et al., 1990).

Individual muscle fiber cross-sectional area (fiber "size") was determined from camera lucida drawings using the bit pad. Fiber areas were measured in the same three sections used to calculate fiber number (see below). Four animals from each treatment group were selected: male DHT treated and untreated and female DHT treated and untreated (16 animals in total). Sixty fibers were measured from innervated and denervated sides of each larynx ( 20 fibers per section); the experimenter was not aware of the state of innervation of the muscle at the time of measurement. There is a wide range of fiber sizes within the larynx. Frequency histograms of fiber size were examined for every animal (examples are shown in Fig. 4). Fibcr sizc is normally distributcd, and therefore the mean is an accurate measure of central tendency. The mean and standard deviation of fiber size for each muscle were obtained.

The number of fibers in each larynx was determined as described in Marin et al. (1990). From the three sections described above, a rectangular area $\left(8825 \mu \mathrm{m}^{2}\right)$ within the inner leaf of the bipennate muscle just dorsal and slightly lateral to the thyrohyals was chosen, and fibers were counted within this region. Fiber number for each hemisection was determined by multiplying the number of fibers per square micrometer by the total area. These values were multiplied by 2 to facilitate comparison with previous results that examined fiber number in whole larynx (Marin et al., 1990). Six larynges from each treatment group were used.

Nerve and muscle ultrastructure. Additional thin sections $(80 \mathrm{~nm})$ were obtained from the Epon-cmbedded larynges described above and examined with an electron microscope (JEOL 1200 EX). Matching areas $\left(0.5-1.0 \mathrm{~mm}^{2}\right)$ of laryngeal muscle from the innervated and denervated sides of four male and three female untreated and two male and two female DHT-treated frogs were examined; one section from each larynx was used. Sections were stained with uranyl acetate and lead citrate. 

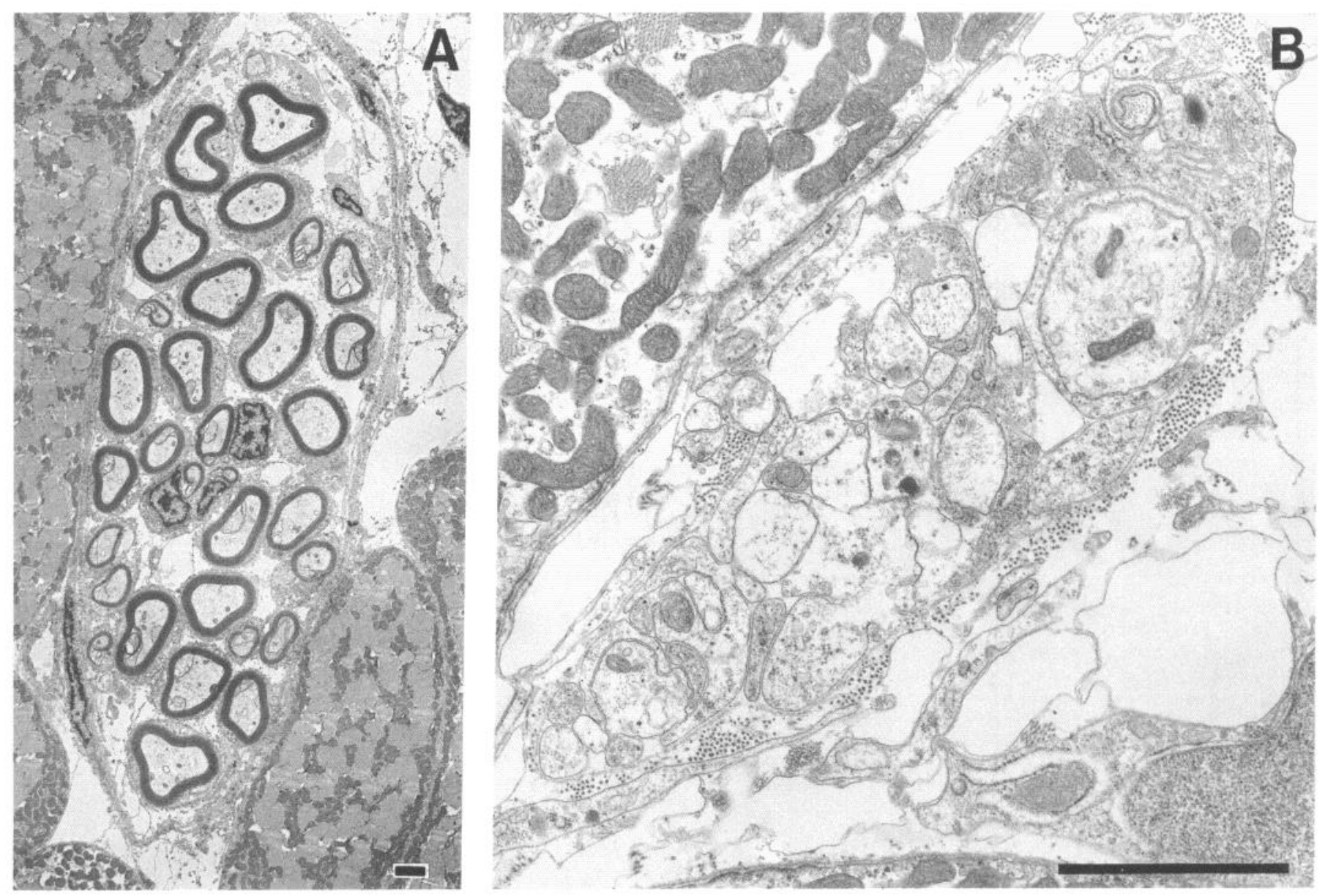

Figure 1. Electron micrographs of nerve $(A)$ and nerve remnant $(B)$ in innervated and denervated laryngeal muscle. The intact nerve contains well-defined axons surrounded by myelin. The degenerating nerve contains no myelinated axons. Scale bars, $2 \mu \mathrm{m}$.

\section{Data analysis}

For each of the dependent measures (number and size of laryngeal muscle fibers), a repeated-measures analysis of variance (ANOVA) was performed, using one within-subject factor (innervation/denervation) and two between-subject factors (sex and DHT treatment). For each dependent measure, the effects of-and the interactions between-innervation, sex, and treatment were examined. If a significant effect of sex on a dependent measure was present, then post hoc $t$ tests were used to determine which sex was more affected.

\section{Results}

\section{Nerve ultrastructure}

No indication of reinnervation in the denervated halves of laryngeal muscle was present at death. The consequences of surgical denervation were further assessed by comparing denervated and innervated sides of larynges in the electron microscope. Bundles of myelinated axons are prominent in sections through innervated laryngeal muscle (Fig. $1 \mathrm{~A}$ ). No myelinated axons are present in denervated laryngeal muscle. Degenerating, unmyelinated axons are present in some denervated muscles; the nerve is markedly atrophic (Fig. $1 B$ ). No signs of reinnervation in denervated muscle were seen in the electron microscope. We conclude that at 4 weeks after denervation there are no healthy axons in laryngeal muscle.

\section{Fiber twitch type}

Juvenile male and female laryngeal muscle contains a mixed population of slow- and fast-twitch fibers in innervated and denervated muscle (Fig. 2). With the acid-stable ATPase method, the small, darkly staining fibers are slow and the large, lightly staining fibers are fast (Sassoon et al., 1987). There is no effect of denervation on the composition of fibers in male or female laryngeal muscle; the percentage of slow-twitch fibers is the same on the innervated and denervated sides ( $p>0.05$; Fig. 3$)$. DHT treatment completely converts both innervated and denervated laryngeal muscle to all fast twitch (Fig. 2); there are no slowtwitch fibers in androgen-treated male or female laryngeal muscle (Fig. 3).

We conclude that androgen-induced conversion of muscle fiber twitch type in laryngeal muscle does not rely on innervation. Since the percentage of slow-twitch fibers was the same in untreated innervated and denervated muscle, denervation by itself does not affect the relative proportion of slow-twitch fibers. There was no sex difference in the effects of denervation or androgen treatment on fiber type composition.

\section{Muscle fiber size}

Denervation results in atrophy and DHT treatment in hypertrophy of male and female laryngeal muscle fibers (Fig. 4). The range of fiber sizes is broad $\left(25-225 \mu \mathrm{m}^{2}\right.$ for untreated animals, $30-450 \mu \mathrm{m}^{2}$ for DHT-treated animals) but approximates a normal distribution. In both untreated and DHT-treated larynges, the mode and mean of the denervated fibers are shifted to the left (fiber area decreases). In both innervated and denervated larynges, DHT treatment causes an overall shift to the right 

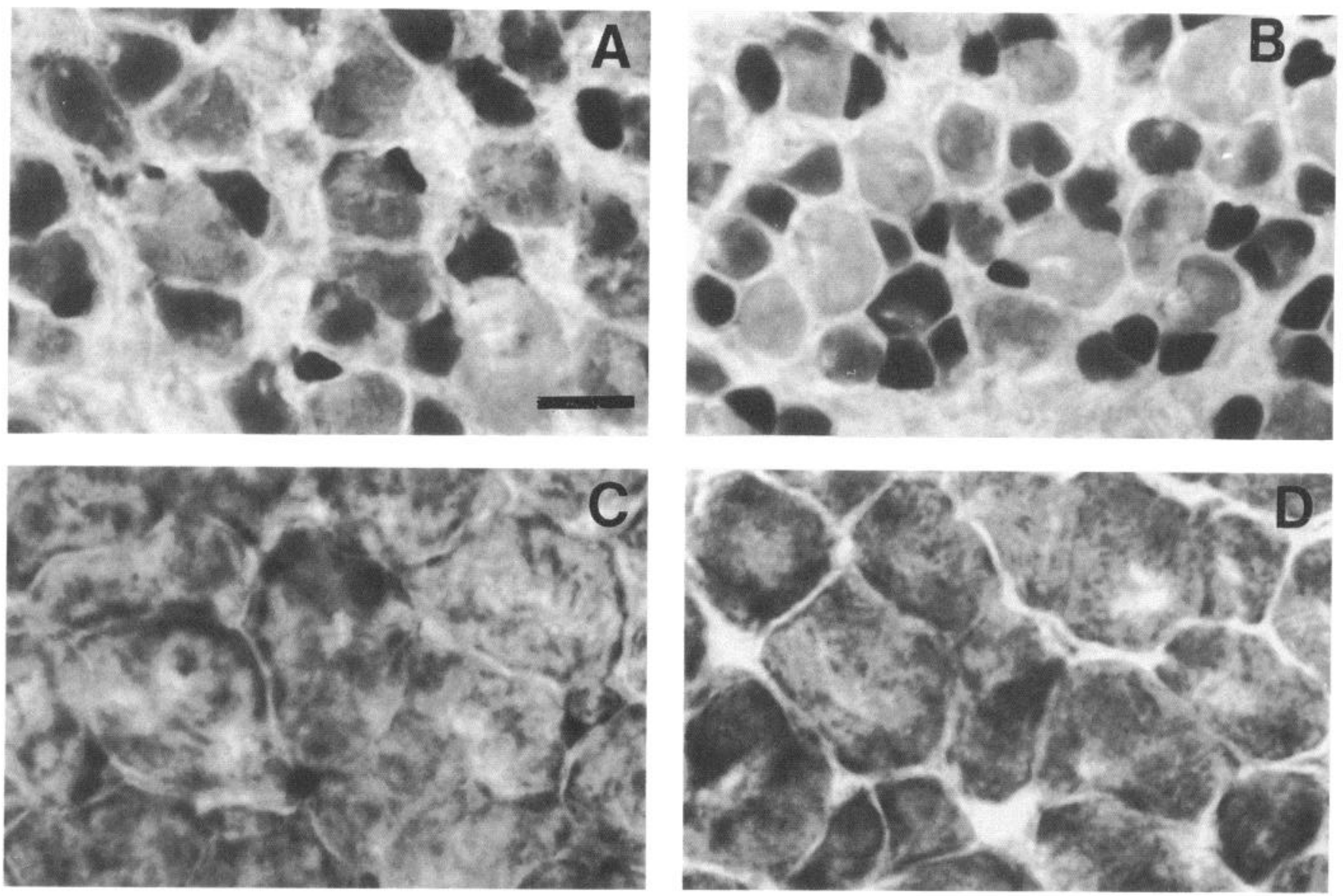

Figure 2. ATPase histochemistry in male laryngeal muscle. $A$ and $B$, Untreated muscle from the innervated $(A)$ and denervated $(B)$ sides of the same juvenile male 4 weeks after denervation. $C$ and $D$, DHT-treated muscle from the innervated $(C)$ and denervated $(D)$ sides of the same male 4 weeks after treatment and denervation. Identical results were observed in female laryngeal muscle. Scale bar, $20 \mu \mathrm{m}$.

(fiber area increases) as well as a broadening of the distribution of fiber sizes. The effect of DHT treatment is less pronounced in denervated fibers.

Denervated muscle fibers are smaller than innervated muscle fibers in both untreated and treated muscle (innervated vs denervated, $p<0.005 ;$ Fig. 5). DHT treatment induces significant hypertrophy of both denervated and innervated laryngeal muscle fibers (untreated vs DHT treated, $p<0.0005$ ). Thus, at the level of individual fibers, androgen-induced growth can occur even in the absence of the motor nerve. There is no sex difference in fiber size regardless of state of innervation or hormone treatment $(p>0.1)$. The effects of denervation and DHT treatment on fiber size are independent $(p>0.1)$. In addition, there is no sex difference in the effects of denervation $(p>0.2)$ or DHT treatment $(p>0.1)$. There is no significant three-way interaction among sex, denervation, and DHT treatment $(p>0.6)$.

\section{Laryngeal muscle fiber number}

We next examined effects of denervation and androgen treatment on laryngeal muscle fiber number (Fig. 6). In untreated males, muscle fiber number is significantly lower on the denervated side $(p<0.005$, paired $t$ test). In untreated females, however, fiber number is not significantly different between the innervated and denervated sides. DHT treatment produces a significant increase in fiber number on the innervated, but not on the denervated, side of the larynx in females (paired $t$ test, $p<0.005$ ). In DHT-treated males, fiber number on the inner-

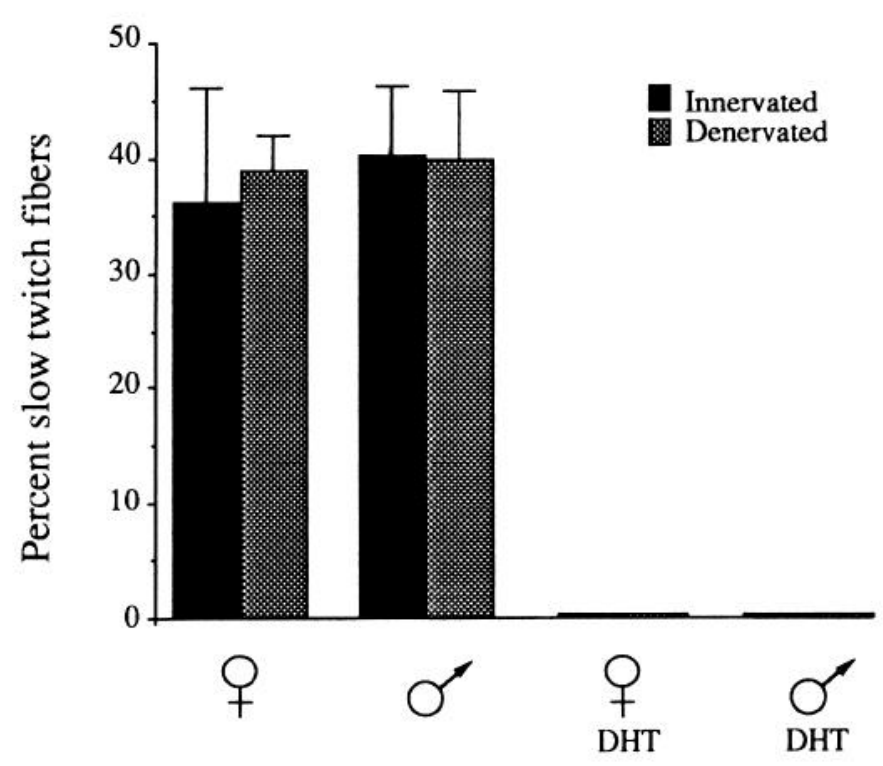

Figure 3. The percentage of slow-twitch fibers in untreated and DHTtreated male and female larynges. DHT treatment converts all fibers to fast twitch; there are no slow-twitch fibers. There is no difference in the percentage of slow-twitch fibers between innervated and denervated sides or between males and females. Each bar indicates the mean and SD for six animals. 
Innervated

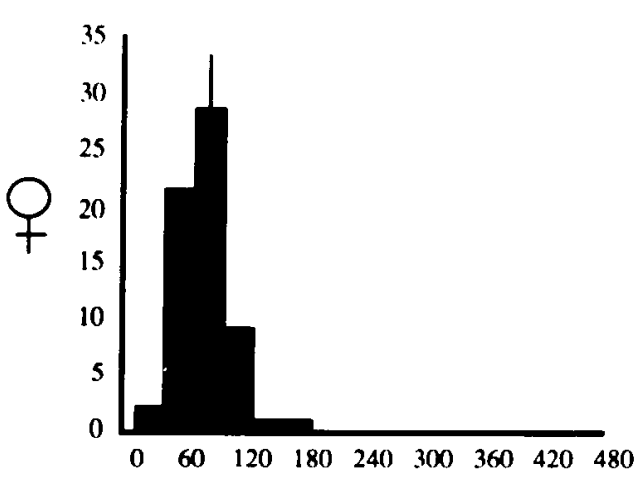

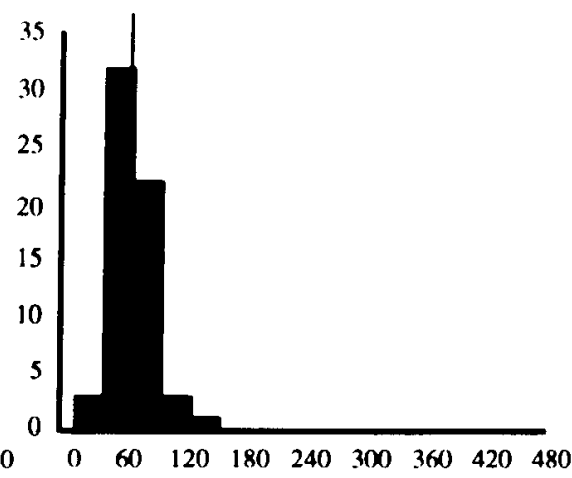

Denervated

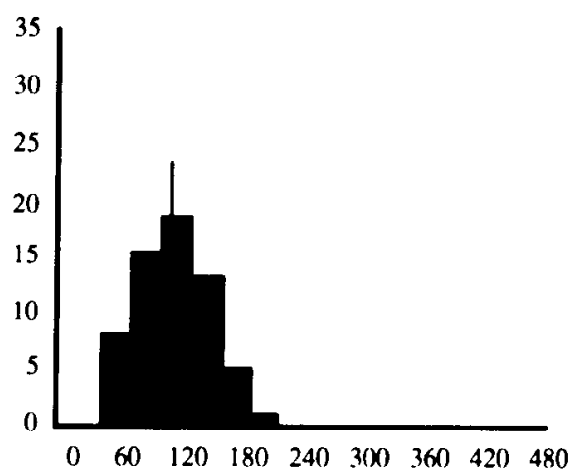

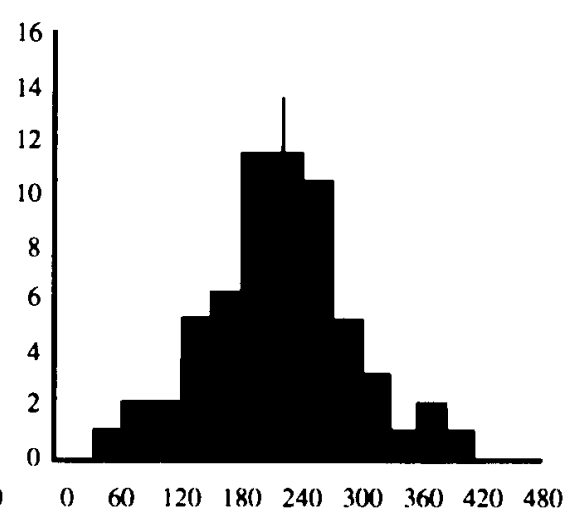

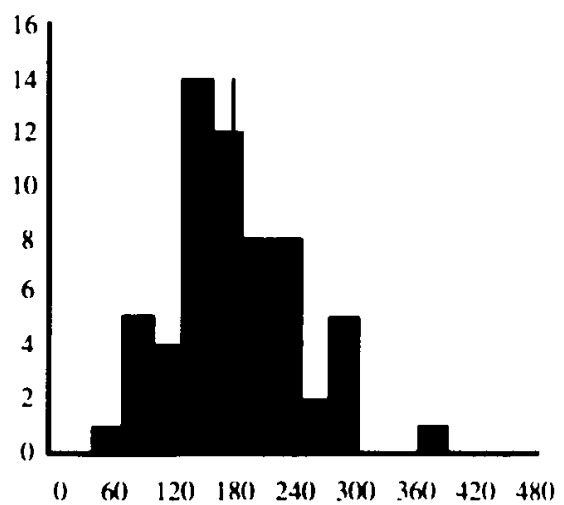

$\begin{array}{lllllllll}0 & 60 & 120 & 180 & 240 & 300 & 360 & 420 & 480\end{array}$
Figure 4. Frequency histograms of fiber sizes in male and female untreated and DHT-treated larynges. For a representative frog from each group, fiber cross-sectional areas $\left(\mu \mathrm{m}^{2}\right)$ of 60 fibers from the innervated and denervated half of the larynx are illustrated. The mean is indicated by the vertical line.

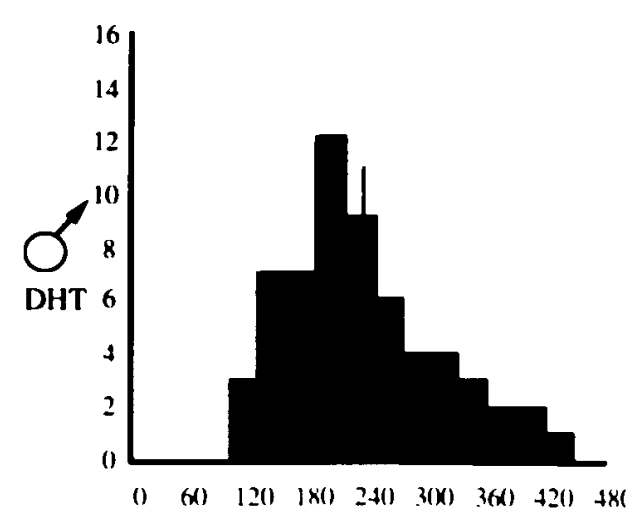




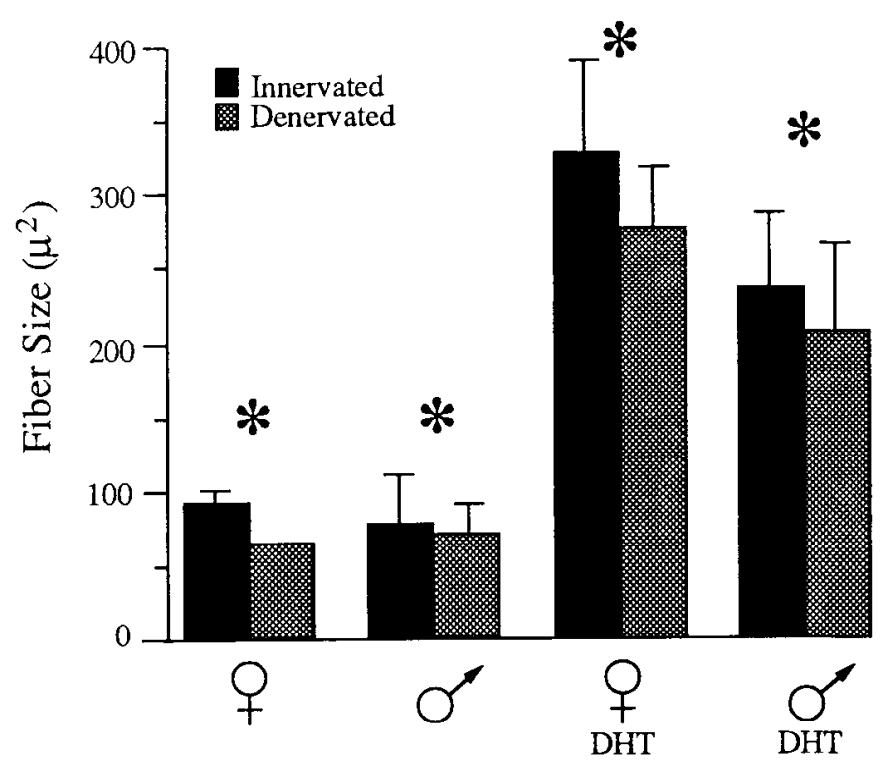

\begin{tabular}{l|c|c|c} 
& Sex & Denervation & DHT treatment \\
\hline Sex & $\mathrm{P}>1$ & $\mathrm{P}>.2$ & $\mathrm{P}>.1$ \\
\hline Denervation & & $\mathrm{P}<.005$ & $\mathrm{P}>.1$ \\
\hline DHT treatment & & & $\mathrm{P}<.0005$ \\
\hline
\end{tabular}

Three way interaction, analysis of variance; $P>6$

Figure 5. Laryngeal muscle fiber size in untreated and DHT-treated male and female larynges. Asterisks indicate significant differences. The mean and SD for four animals from each group are illustrated (top). Results of an ANOVA reveal significant effects of each independent variable (sex, denervation, DHT treatment) on the diagonal (bottom), and their pairwise interactions above the diagonal. The three-way interaction was not significant.

vatcd side of the larynx does not differ significantly from that on the denervated side (innervated vs denervated, $p>0.05$ ). Since androgen treatment results in similar fiber number on the innervated and denervated sides of male larynx, it is likely that DHT compensates for the loss of innervation.

Thus, sex, denervation, and DHT treatment all have a significant effect on laryngeal muscle fiber number (Fig. 6). The effect of denervation is sexually dimorphic $(p<0.01)$. Iowever, the effect of DHT treatment is the same in the sexes $(p>0.6)$. The effects of denervation and DHT treatment on fiber number are independent $(p>0.4)$. Because the effect of DHT treatment varies with both innervation and sex, there is a significant threeway interaction between sex, denervation, and DHT treatment $(p<0.03)$.

Laryngeal muscle was examined with the electron microscope to determine the size of the smallest fibers and to examine the muscle for degenerating fibers (Fig. 7). Because fiber size decreases in denervated muscle, we were concerned that some fibers might atrophy to a size below the resolution of the light microscope and therefore be excluded from fiber counts. The smallest fibers observed in the electron microscope were approximately $2 \mu \mathrm{m}$, which is large enough to be detected in the light microscope. Thus, our fiber counts probably include every fiber. We wished to determine if the lower fibcr number observed in denervated, but not innervated, male laryngeal muscle was due to fiber degeneration. No evidence of fiber degeneration was present in DHT-treated muscle (innervated or denervated) or in innervated muscle (male or female). However, in untreat-

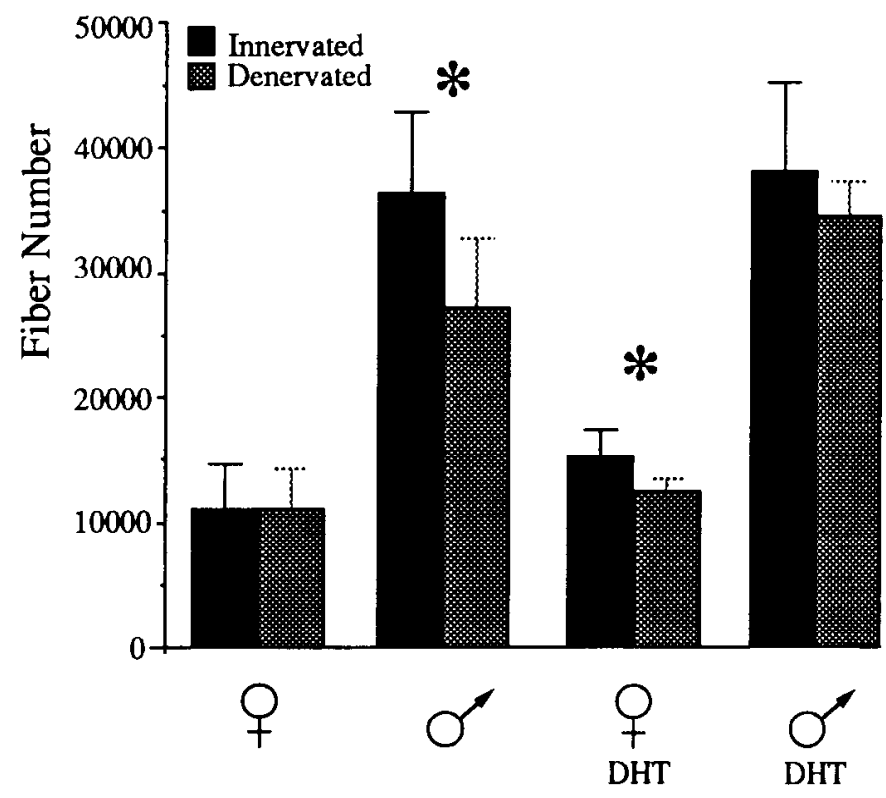

\begin{tabular}{l|c|c|c} 
& Sex & Denervation & $D H T$ treatment \\
\hline Sex & $P<.0005$ & $P<.01$ & $P>.6$ \\
\hline Denervation & & $P<.0005$ & $P>.4$ \\
\hline DHT treatment & & & $P<.05$ \\
\hline
\end{tabular}

Three way interaction, analysis of variance; $P<.03$

Figure 6. Laryngeal muscle fiber number in untreated and DHT-treated male and female larynges. Asterisks indicate significant differences. The mean and SD for six animals from each group are illustrated (top). Results of an ANOVA reveal significant effects of each independent variable (sex, denervation, DHT treatment) on the diagonal (bottom), and their pairwise interactions above the diagonal. The three-way interaction was significant.

ed, denervated muscle, degenerating fibers were present in both sexes; examples of such fibers in male denervated muscle are illustrated (Fig. 7). We conclude that the decreased number of muscle fibers in denervated male laryngeal muscle is due to fiber necrosis. Because degenerating fibers were also present in female muscle, we conclude that fiber loss is also occurring in females but cither is of less magnitude or is slower than the comparable process in males.

\section{Discussion}

Conversion of fiber twitch type is androgen dependent and nerve independent; the effects of denervation and DHT treatment are the same in males and females

In Xenopus laevis, androgen secretion is responsible for the developmental transformation of muscle fiber types in male laryngeal muscle from mixed fast- and slow-twitch to entirely fast-twitch (Sassoon et al., 1987; Tobias et al., 1991b). Neither females nor males castrated before PM2 ever undergo fiber transformation. Previous studies have shown that androgen treatment in juvenile frogs can convert laryngeal muscle to all fast-twitch (Sassoon et al., 1987; Tobias et al., 1991b). One aim of this study was to determine whether innervation provided by the motor nerve is required for androgen-induced fiber transformation. The answer is clearly no. Denervation has no effect on DHT-induced fiber transformation in male or female laryngeal muscle.

A similar result has been found in developing rat hindlimb 

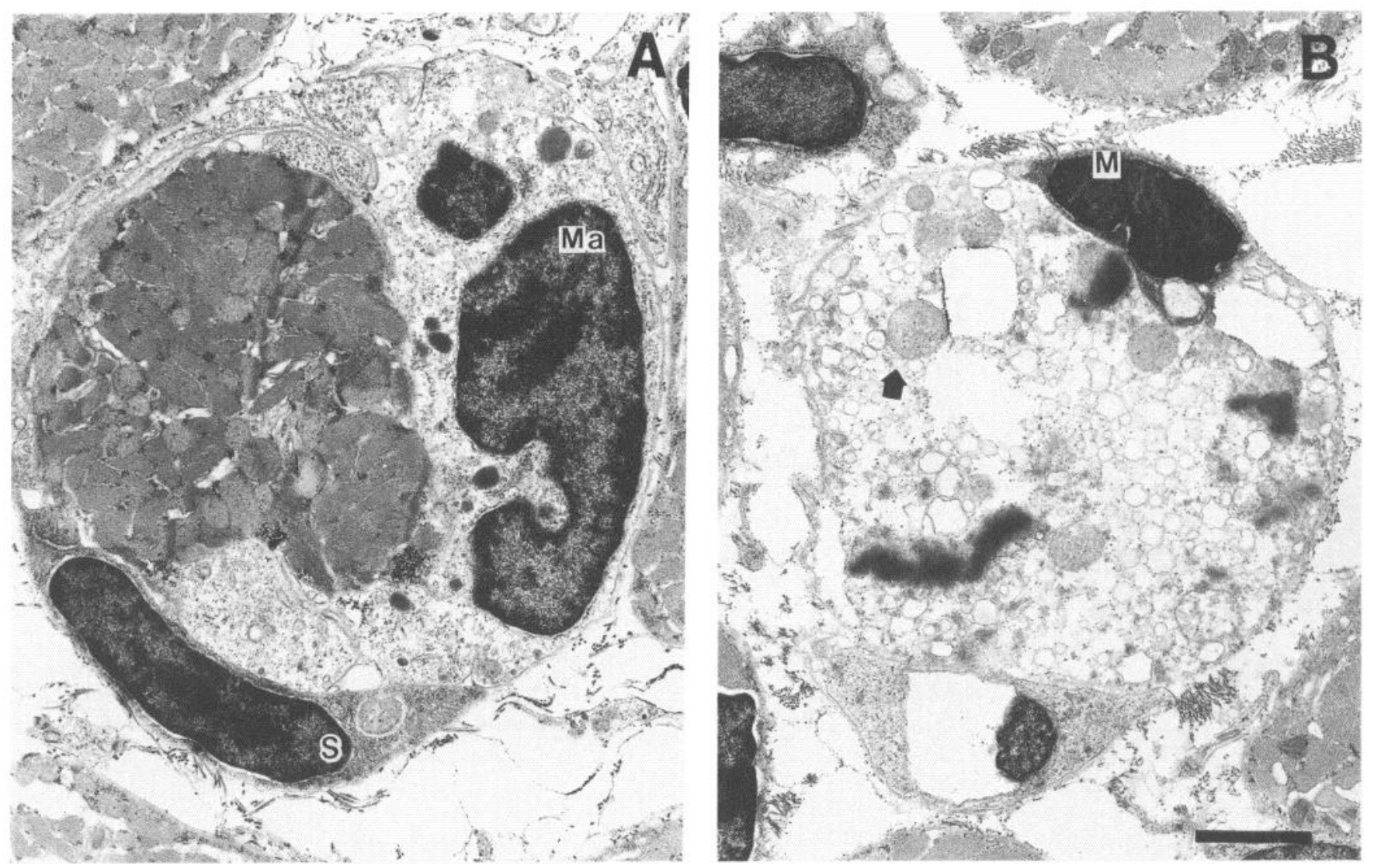

Figure 7. Electron micrographs of degenerating muscle fibers in a male denervated laryngeal muscle. $A$, Degenerating muscle fiber engulfed by a macrophage $(M a)$. The cell at lower left $(S)$ has the morphological characteristics of a satellite cell. $B$, Degenerating fiber undergoing necrosis; note the condensed chromatin in the myonucleus $(M)$, large vacuoles, and distended mitochondria (arrow). Scale bar, $2 \mu \mathrm{m}$.

muscle in which thyroid hormone treatment induces precocious adult myosin expression in both innervated and denervated muscle (Russell et al., 1988). A large body of evidence in other muscles, however, suggests that innervation can strongly influence muscle fiber type (reviewed in Jolesz and Streter, 1981). To what extent is the neural independence of fiber type expression a special property of these hormone-sensitive muscles? Until quite recently, in vivo and in vitro studies on chick and rodent muscle suggested that the earliest-appearing muscle fibers, primary myotubes, are nerve independent; primary myofibers form and express characteristic myosins without innervation (Butler et al., 1982; Crow and Stockdale, 1986; Hoh et al., 1989). Secondary myotubes, which arise subsequently and form the bulk of fibers in adult muscles, were thought to require innervation for proliferation and survival in vivo; this dependence made it difficult to assess whether expression of musclespecific fiber types requires innervation (Harris, 1981; McLennan, 1983; Ross et al., 1987). A reinvestigation of this issue in chick limb has revealed a population of secondary myofibers that survive the absence of the nerve or its activity (Fredette and Landmesser, 1991). These secondary myotubes express characteristic fiber types in the absence of innervation. Aneural muscle of embryonic rat hindlimbs has also been shown to express stage-appropriate myosins (Condon et al., 1990). Thus, recent experiments suggest that the nerve is not required for expression of muscle-appropriate myosins in developing muscle. Hormone-sensitive muscles, unlike other skeletal muscles, do depend on endocrine factors for expression of adult myosins.
The mechanism by which hormones induce fiber switching is not yet clear. Male larynges undergo a rapid period of muscle fiber addition during early postmetamorphic development (Marin et al., 1990). Conversion from mixed slow and fast to all fast fibers could involve either switching myosin expression in existing fibers or the death of slow fibers and their replacement by newly formed fast fibers. We currently favor the switching hypothesis because muscle fiber number is nearly adult at the time when laryngeal muscle conversion begins (Marin et al., 1990) and, as we describe here, no signs of fiber degeneration are observed in innervated developing male muscle. Treatment with DHT also induces an all fast-twitch complement of fibers without any evidence of fiber degeneration. Thus, androgeninduced fiber switching is probably due to conversion of slowtwitch fibers to fast-twitch fibers.

Because these experiments required rapid switching of muscle fiber type accomplished through exposure to supraphysiological levels of androgen, we cannot rule out the possibility that the androgen-sensitive motor neuron contributes to fiber conversion during the 6 month period in which fiber type normally masculinizes. However, androgen-induced muscle fiber conversion in denervated muscle strongly suggests that androgen can act directly on the muscle to induce transformation of twitch type. Fiber type conversion could be accomplished via DHTregulated expression of a fast-twitch myosin. We have recently cloned a laryngeal-specific myosin heavy chain gene from the $X$. laevis larynx (Catz et al., 1992). Expression of this gene is sexually differentiated and androgen-regulated as is fiber twitch 
type, and could be responsible for androgen-regulated muscle fiber type conversion.

Fiber size is affected by innervation and androgen; there is no sex difference in the effects of denervation and DHT treatment on fiber size

Denervation produces atrophy and androgen produces hypertrophy in male and female laryngeal muscle fibers. The effects of hormone treatment and state of innervation are independent and additive. Effects are independent because DHT increases muscle fiber size in both denervated and innervated larynges and because denervation decreases muscle fiber size in both untreated and hormone-treated larynges. Effects are additive because the combined effect of innervation plus DHT treatment is greater than the effect of either alone.

Since both laryngeal muscle and motor neurons express androgen receptor at this stage of development, as do neurons in most brain nuclei of the "calling pathway" (Gorlick and Kelley, 1986; Kelley et al., 1989), androgen could be acting either on the muscle directly or via the motor neurons to produce fiber hypertrophy. Our data show that androgen can produce larger muscle fibers directly, without the presence of the motor nerve, although maximal hypertrophy requires innervation. It is not clear whether innervation per se, as opposed to innervation by androgen receptor-expressing motor neurons, is specifically required for achievement of maximal fiber size. Cross-innervation by non-androgen receptor-expressing motor neurons could address this question.

Effects of androgen and denervation have also been examined in a sexually dimorphic muscle of rats, the levator ani. In young adult rats, testosterone treatment increases muscle weight in innervated but not in denervated levator ani (Godinho et al., 1987b), suggesting that an intact nerve supply is required for maximal growth. Muscle atrophy in response to castration or denervation is regulated somewhat differently: castration produces greater atrophy than denervation and combined effects do not exceed those of castration alone (Godinho et al., 1987a). In older males, however, denervation and castration do produce greater atrophy of levator ani than either treatment alone (Araki et al., 1991). Muscle size in levator ani is influenced by both androgen and innervation as in $X$. laevis larynx; whether effects are additive may depend on the age of the animal.

$X$. laevis larynx differs from rat levator ani in that muscle size is not androgen dependent in adulthood (Segil et al., 1986; Watson and Kelley, 1992). In juveniles, castration halts but does not reverse the progress of masculinization (Marin et al., 1990; Tobias et al., 1991b). This species difference may reflect the opportunistic breeding strategy used by $X$. laevis but not by rats. In $X$. laevis, bouts of courtship song and spawning activity are rapidly triggered during a prolonged breeding season by rainfall and subsequent drops in temperature (Kalk, 1960). Involution of laryngeal muscle between spawning bouts would place individual males at a competitive disadvantage in attracting females.

The effects of denervation are mediated by a reduction in the activity of muscle fibers; atrophy can be induced by blocking neuromuscular transmission and prevented by direct stimulation of the muscle (Goldspink, 1980). Muscle activity may also be involved in androgen regulation of fiber size. In a related androgen-sensitive system, the clasper motor neurons of $X$. lae$v i s$, androgen increases excitability and castration decreases excitability (Erulkar et al., 1981). In laryngeal myotubes cultured from adult myoblasts, androgen has been shown to increase the conductances and mean channel open times of ACh receptors (Erulkar and Wetzel, 1989). It is thus possible that the additive effects of androgen and innervation on laryngeal muscle fiber size reflect increases in the activity of laryngeal motor neurons and in the excitability of laryngeal muscle fibers.

\section{Fiber number depends on sex, innervation, and androgen}

Denervation causes a significant decrease in laryngeal fiber number in male frogs. A difference in fiber number between the innervated and denervated sides of male larynges could be due either to fiber loss on the denervated side or to fiber addition only on the innervated side. The first alternative implies that the nerve is required for the maintenance of existing fibers and that denervation results in cell death. The second alternative implies that the nerve is required for ongoing fiber addition and that the decrease in fiber number on the denervated side represents failure to add new fibers. Two lines of evidence favor the first alternative. The rate of fiber proliferation has been determined in male and female larynges throughout postmetamorphic development (Marin et al., 1990). At the stage when the larynx was denervated, fiber proliferation is slow in males. The number of fibers added during the 4 week period of denervation $(\sim 1000)$ is considerably less than the observed difference between the innervated and denervated sides $(\sim 10,000$ fibers). Thus, the difference in fiber number between the innervated and denervated sides cannot be due solely to fiber addition on the innervated side. Furthermore, degenerating fibers were observed in denervated muscle while no degenerating fibers were found in innervated muscle. Together, these data suggest that the nerve is required for maintenance of existing fibers and denervation results in fiber death.

Fiber number is similar on the innervated and denervated sides of DHT-treated male larynges. Thus, DHT treatment compensates for denervation-induced fiber loss in males. Although the ultimate effect of innervation or DHT treatment on fiber number is the same, it is not clear if they act through the same mechanism. For example, DHT might induce fiber proliferation in denervated muscle rather than maintain existing fibers on the denervated side. It is difficult to distinguish between these possibilities since fibers were counted long after ( 4 weeks) the nerve was severed. However, no degenerating fibers were observed in DHT-treated, denervated muscle, suggesting that DHT, like the nerve, maintains existing frbers. Also, there is no difference in fiber number on the innervated side of untreated or DHT-treated larynges, suggesting that DHT does not induce fiber proliferation in juvenile males.

Denervated male larynges lose nearly one-third of their fibers. Denervated female larynges do not lose fibers although ultrastructural studies indicate that female larynges are undergoing some fiber degeneration. These data suggest that male laryngeal muscle is more sensitive to denervation than is female laryngeal muscle. A possible explanation for this sex difference in fiber loss is that newly formed fibers, or myotubes, are more susceptible to denervation than are older fibers. Our fiber counts include both myofibers and myotubes, as these cannot be distinguished in the light microscope (Marin et al., 1990). Fiber addition prior to PM2 is very rapid in male larynx (approximately 25,000 fibers are added) and slow in female larynx (approximately 3000 fibers are added; Marin et al., 1990). Thus, there are many more newly added fibers in males than in females at the stage when the larynx was denervated. 
In a previous experiment, testosterone treatment-induced fiber addition in the female larynx at PM0 but not at PM2 (Marin et al., 1990). In this study, however, fiber addition was induced by DHT treatment in PM2 females. DHT may be a more myogenic androgen or females may convert testosterone to estrogen, a nonmyogenic steroid in larynx. Does androgen-induced fiber addition in females, but not in males, suggest that there is a different population of myoblasts in the sexes? Not necessarily. Androgen-induced fiber addition in males ends at PM2; males castrated at PM2 continue to add fibers slowly, as do intact PM2 males (Marin et al., 1990). Androgen-sensitive myoblasts in the male may be depleted by PM 2 due to prior androgen-induced myogenesis while androgen-sensitive myoblasts in the female are still available for stimulation by exogenous androgen.

Fiber addition in females is only observed on the innervated side, suggesting that hoth androgen and innervation are required. We do not know if innervation is also required for androgen-induced fiber addition in male larynx. If so, androgen could either affect the muscle directly or affect the muscle indirectly via the nerve. Some laryngeal myoblasts may require both a neural trophic factor as well as androgen for proliferation. Alternatively, androgen might act only on the motor neurons to induce myoblast proliferation.

In summary, we have used the androgen-sensitive laryngeal muscle of Xenopus laevis to study the relative contributions of innervation and hormone exposure to three muscle properties: fiber type, size, and number. In addition, we have examined the effects of denervation and DHT treatment on males and females to determine if there is a sex difference in sensitivity to these treatments. Fiber typc conversion relies solely on androgen exposure. Innervation is neither necessary nor sufficient to effect fiber type conversion; denervation has no effect on the proportion of fiber types in the larynx. Androgen is also a more powerful determinant of muscle fiber size than innervation; androgen-treated fibers are larger than untreated fibers whether they are innervated or not. However, denervation reduces the affects of androgen on fiber growth. These results suggest that innervation and androgen effects on fiber size are independent and additive. Fiber number is regulated by the interaction of nerve and hormone. Androgen-induced fiber addition has only been observed in innervated female muscle. However, androgen treatment can compensate for denervation-induced fiber loss in males. These data suggest that androgen can affect fiber type, size, and maintenance of fiber number at the level of the muscle fiber directly and not via the nerve. The nerve has no effect on fiber type, affects fiber size independently of hormone, and together with hormone affects fiber number.

\section{References}

Araki I, Harada Y, Kuno M (1991) Target-dependent hormonal control of neuron size in the rat spinal nucleus of the bulbocavernosus. J Neurosci 11:3025-3033.

Breedlove SM, Arnold AP (1983) Hormonal control of a developing neuromuscular system: I. Complete demasculinization of the spinal nucleus of the bulbocavernosus in male rats using the anti-androgen, flutamide. J Neurosci 3:417-423.

Butler J, Cosmos E, Brierley J (1982) Differentiation of muscle fiber types in aneurogenic brachial muscles of the chick embryo. J Exp Zool 224:65-80.

Catz D, Fischer L, Moschella T, Tobias ML, Kelley DB (1992) Sexually dimorphic expression of a laryngeal-specific, androgen-regulated myosin heavy chain gene during Xenopus laevis development. Dev Biol $154: 368-378$.

Condon K, Silberstein L, Blau HM, Thompson W (1990) Differen- tiation of fiber types in aneural musculature of the prenatal rat hindlimb. Dev Biol 138:275-295.

Crow MT, Stockdale FE (1986) Myosin expression and specialization among the earliest muscle fibers of the developing avian limb. Dev Biol 113:238-254.

Erulkar S, Kelley D, Jurman M, Zemlan F, Schneider G, Krieger (1981) The modulation of the neural control of the clasp reflex in male Xenopus laevis by androgens. Proc Natl Acad Sci USA 78:5876-5880.

Erulkar SD, Wetzel DM (1989) 5 Alpha dihydrotestosterone has nonspecific effects on membrane channels and possible genomic effects on ACh-activated channels. J Neurophysiol 61:1036-1052.

Fischer L, Kelley DB (1991) Androgen receptor expression and sexual differentiation of effectors for courtship song in Xenopus laevis. Semin Neurosci 3:469-480.

Fredette B, Landmesser L (1991) A reevaluation of the role of innervation in primary and secondary myogenesis in developing chick muscle. Dev Biol 143:19-35.

Godinho RO, Lima-Landman MT, Souccar C, Lapa AJ (1987a) Trophic control of cholinesterase activity in a testosterone-dependent muscle of the rat: effects of castration and denervation. Exp Neurol 96:558-568.

Godinho RO, Lima-Landman MT, Souccar C, Lapa AJ (1987b) Trophic control of cholinesterase activity in a testosterone-dependent muscle of the rat: effects of testosterone administration. Exp Neurol 98:93-102.

Goldspink D (1980) Physiological factors influencing protein turnover and muscle growth in mammals. In: Development and specialization of skeletal muscle (Goldspink D, ed), pp 65-89. Cambridge: Cambridge UP.

Gorlick D, Kelley DB (1986) The ontogeny of androgen receptors in the CNS of Xenopus laevis. Dev Brain Res 26:193-201.

Guth L, Samaha F (1970) Procedure for histochemical demonstration of actomyosin ATPase. Exp Neurol 28:365-367.

Harris AJ (1974) Inductive functions of the nervous system. Annu Rev Physiol 36:251-305.

Harris AJ (1981) Embryonic growth and innervation of rat skeletal muscles. I. Neural regulation of muscle fibre numbers. Philos Trans R Soc Lond [Biol] 293:257-277.

Hoh JFY, Hughes S, Hugh G, Porgaj I (1989) Three hierarchies in skeletal muscle fibre classification allotype, isotype and phenotype. In: Cellular and molecular biology of muscle development (Kedes LI, Stockdale FE, eds), pp 150-26. New York: Liss.

Izumo S, Nadal-Ginard B, Mahdavi V (1986) All members of the MHC multigene family respond to thyroid hormone in a highly tissuespecific manner. Science 231:597-600.

Jolesz F, Streter FA (1981) Development, innervation, and activitypattern induced changes in skeletal muscle. Annu Rev Physiol 43: $531-552$.

Kalk M (1960) Climate and breeding in Xenopus laevis. S Afr J Med Sci 11:271-276.

Kelley DB, Sassoon D, Segil N, Scudder M (1989) Development and hormone regulation of androgen receptor levels in the sexually dimorphic larynx of Xenopus laevis. Dev Biol 131:111-118.

Lyons GE, Kelly AM, Rubinstein NA (1986) Testosterone-induced changes in contractile protein isoforms in the sexually dimorphic temporalis muscle of the guinea pig. J Biol Chem 261:13278-13284.

Marin ML, Tobias ML, Kelley DB (1990) Hormone-sensitive stages in the sexual differentiation of laryngeal muscle fiber number in Xenopus laevis. Development 1 10:703-712.

McLennan IS (1983) Neural dependence and independence of myotube production in chicken hindlimb muscles. Dev Biol 98:287-294.

Koss JJ, Duxson MJ, Harris AJ (1987) Neural determination of muscle fibre numbers in embryonic rat lumbrical muscles. Development 100: 395-409.

Rubinstein NA, Erulkar SD, Schneider GT (1983) Sexual dimorphism in the fibers of a "clasp" muscle of Xenopus laevis. Exp Neurol 82: $424-431$

Russell SD, Cambon N, Nadal-Ginard B, Whalen RG (1988) Thyroid hormone induces a nerve-independent precocious expression of fast myosin heavy chain mRNA in rat hindlimb skeletal muscle. J Biol Chem 263:6370-6374.

Sassoon D, Kelley DB (1986) The sexually dimorphic larynx of Xenopus laevis: development and androgen regulation. Am J Anat 177: $457-472$.

Sassoon D, Gray G, Kelley DB (1987) Androgen regulation of muscle 
fiber type in the sexually dimorphic larynx of Xenopus laevis. J Neurosci 7:3198-3206.

Scgil N, Silverman L, Kelley D (1986) Androgen-binding levels in a sexually dimorphic muscle of Xenopus laevis. Gen Comp Endocrinol 66:95-101.

Tobias ML, Marin ML, Kelley DB (1991a) Development of functional sex differences in the larynx of Xenopus laevis. Dev Biol 147:251-259.
Tobias ML, Marin ML, Kelley DB (1991b) Temporal constraints on androgen directed laryngeal masculinization in Xenopus laevis. Dev Biol 147:260-270.

Watson J, Kelley D (1992) Testicular masculinization of vocal behavior in juvenile female Xenopus laevis reveals sensitive periods for song duration, rate and frequency spectra. J Comp Physiol [B] 171: 343-350. 